\title{
THE ETA INVARIANT AND FAMILIES OF PSEUDODIFFERENTIAL OPERATORS
}

\author{
Richard B. MELRose
}

\begin{abstract}
For a compact manifold without boundary a suspended algebra of pseudodifferential operators is considered; it is an algebra of pseudodifferential operators on, and translation-invariant in, an additional real variable. It is shown that the eta invariant, as defined by Atiyah, Patodi and Singer for admissible Dirac operators, extends to a homomorphism from the ring of invertible elements of the suspended algebra to the additive real line. The deformation properties of this extended eta homomorphism are discussed and a related 'divisor flow' is shown to label the components of the set of invertible elements within each component of the elliptic set.
\end{abstract}

\section{Introduction}

The eta invariant of the spin Dirac operator, and of the signature operator, on an odd dimensional manifold was introduced by Atiyah, Patodi and Singer [1] as the boundary correction term for their index formula on an even-dimensional compact manifold with boundary. Their definition extends directly to all 'admissible' Dirac operators and was later shown to extend to all self-adjoint elliptic pseudodifferential operators on compact manifolds without boundary. In the Dirac setting there are various further extensions to non-compact manifolds (by Brüning and Seeley [6], by Müller [16], by Stern [19] and in [13]), to singular manifolds (by Cheeger [8]) to boundary problems (by Branson and Gilkey [5], by Douglas and Wojciechowski [9], by Lesch and Wojciechowski [11] and by Müller [17]), to families (by Bismut and Cheeger [3], [2] and in [14], [15] ) also to define 'higher' eta invariants (by Lott [12], by Getzler [10] and by Wu [20]). Here a somewhat different 'pseudodifferential' extension of the eta invariant is given. This is closely related to Singer's comments in [18] on the formal analogy between the index function and the eta invariant (despite their obvious differences).

Received January 31, 1995.

Supported in part by NSF grant 9306389-DMS.

Manuscript available from ftp-math-papers.mit.edu 
For purposes of comparison consider first the familiar properties of the index. Let $\Psi^{*}(Y)$ be the algebra of 1-step polyhomogeneous pseudodifferential operators on the compact manifold without boundary, $Y$. The element $A$ is elliptic if its symbol $\sigma_{m}(A) \neq 0$ and then it defines a Fredholm operator on $\mathcal{C}^{\infty}(Y)$, i.e., $A: \mathcal{C}^{\infty}(Y) \longrightarrow \mathcal{C}^{\infty}(Y)$ has finite dimensional null space and closed range of finite codimension. The index is the difference of the dimension of the null space and codimension of the range. It is constant on connected components of the set $\operatorname{Ell}_{*}(Y) \subset \Psi^{*}(Y)$ of elliptic elements, which is a ring, and is an additive homomorphism into $\mathbb{Z}$ :

$$
\text { ind }: \operatorname{Ell}_{*} \longrightarrow \mathbb{Z}, \operatorname{ind}(A \circ B)=\operatorname{ind}(A)+\operatorname{ind}(B) \text {. }
$$

Here we consider instead the 'suspended' algebra which we denote by $\Psi_{\text {sus }}^{*}(Y)$. Despite the notation it is a subspace, which is an algebra, of the translation-invariant part of the space $\Psi^{*}(Y \times \mathbb{R})$ of all (1-step polyhomogeneous) pseudodifferential operators on the product $Y \times \mathbb{R}$. This algebra may be thought of as the 'pseudodifferential suspension' of $\Psi^{*}(Y)$. Within it consider the elliptic elements of a given order $\operatorname{Ell}_{m}$, and let $\operatorname{Ell}_{m}(A)$ denote the component containing a given $A \in \operatorname{Ell}_{m}$. Let $\operatorname{Inv}_{m}$ denote the set of invertible elliptic elements, of order $m$.

Although we have simply been discussing pseudodifferential operators acting on functions here we really consider the corresponding algebras of operators acting on sections of a vector bundle, $E$, over $Y$. The central result of this paper is

Theorem 1. For any compact manifold $Y$ and any vector bundle $E$ over $Y$, there is an additive homomorphism $\eta$ from the ring of invertible elliptic elements $\operatorname{Inv}_{*} \subset \Psi_{\text {sus }}^{*}(Y ; E)$ into $\mathbb{C}$, i.e.,

$$
\eta: \operatorname{Inv}_{*} \longrightarrow \mathbb{C}, \eta(A \circ B)=\eta(A)+\eta(B),
$$

and if $\partial$ is an admissible Dirac operator acting on the sections of some Clifford module $E$ over $Y$ then provided $\partial$ is invertible $\eta\left(i D_{t}+ð\right)$ is the eta invariant of $\partial$ in the sense of Atiyah, Patodi and Singer.

Here by an 'admissible' Dirac operator is meant one for which the Clifford module is Hermitian and the connection defining the Dirac operator is Clifford and unitary. The crucial property of such an admissible Dirac operator is embodied in the local index theorem, see the argument of Bismut and Freed [4] or the discussion in [13].

As for the Dirac case, under variation of metric or connection within the class of admissible Dirac operators, the variation of the eta functional 
is local. More precisely there is a function, which is called here the formal trace

$$
\widetilde{\operatorname{Tr}}: \Psi_{\text {sus }}^{*}(Y ; E) \longrightarrow \mathbb{C}
$$

which vanishes for commutators and operators of order less than $-\operatorname{dim} Y$. Thus it only depends on the finite symbols of an operator. It is a suspended analogue of the 'residue trace' of Guillemin and Wodzicki. Similar trace functionals have also been considered by Burns and Mazzeo in work on Hochschild homology, [7].

Theorem 2. If $A_{s} \in \Psi_{\text {sus }}^{m}(Y ; E)$ is a smooth family of elliptic and invertible elements then

$$
\frac{d}{d s} \eta\left(A_{s}\right)=\frac{2}{i} \widetilde{\operatorname{Tr}}\left(\frac{d A_{s}}{d s} A_{s}^{-1}\right)
$$

The fact that $\widetilde{\operatorname{Tr}}(A)$ is local, i.e., symbolic, has the important consequence that $\widetilde{\operatorname{Tr}}\left(\frac{d A_{s}}{d s} A_{s}^{-1}\right)$ can be defined even if $A_{s}$ is not actually invertible but rather is just elliptic. Indeed, replacing $A_{s}^{-1}$ by a parametrix for $A_{s}$ must give the same result. This allows the 'divisor flow' between two invertible elements, $A_{0} \in \operatorname{Inv}_{m}$ and $A_{1} \in \operatorname{Inv}_{m}$ with $A_{1} \in \operatorname{Ell}_{m}\left(A_{0}\right)$, i.e., provided they are in the same component of the elliptic set, to be defined by

$$
\operatorname{DF}\left(A_{1}, A_{0}\right)=\frac{1}{2}\left(\eta\left(A_{1}\right)-\eta\left(A_{0}\right)-\frac{2}{i} \int_{0}^{1} \widetilde{\operatorname{Tr}}\left(\frac{d A_{s}}{d s} B_{s}\right) d s\right)
$$

where $[0,1] \ni s \longmapsto A_{s}$ is a connecting curve in $\operatorname{Ell}_{m}\left(A_{0}\right)$ and $B_{s} \in$ Ell $_{-m}$ is a smooth parametrix, i.e., $A_{s} \circ B_{s}=\mathrm{Id}-R_{s}, R_{s} \in \Psi_{\text {sus }}^{-\infty}(Y ; E)$. As the name is supposed to indicate, this function has properties very similar to the spectral flow for self-adjoint operators.

Theorem 3. For each $A_{0} \in \operatorname{Inv}_{m} \cap \mathrm{Ell}_{m}$ the divisor flow

$$
\operatorname{Ell}_{m}\left(A_{0}\right) \ni A_{1} \longmapsto \operatorname{DF}\left(A_{1}, A_{0}\right) \in \mathbb{Z}
$$

is integer-valued, additive, conjugation-invariant, continuous and distinguishes between (i.e., labels) the connected components of $\operatorname{Inv}_{m} \cap \operatorname{Ell}_{m}\left(A_{0}\right)$.

The proof of these results rests on the definition of a regularized trace functional

$$
\overline{\operatorname{Tr}}: \Psi_{\text {sus }}^{*}(Y ; E) \longrightarrow \mathbb{C} .
$$


The only operator of trace class in $\Psi_{\text {sus }}^{*}(Y ; E)$ is the zero operator. However, if $m<-\operatorname{dim} Y-1$ then this regularized trace functional can be defined by

$$
\overline{\operatorname{Tr}}(A)=\int_{Y} \operatorname{tr} A(y, y, 0)
$$

where the Schwartz kernel $A\left(y, y^{\prime}, t\right)$ is naturally a density on $Y$ and $\operatorname{tr}$ is the trace functional on hom $E$. The extension, (7), defined below still has the fundamental property of vanishing on commutators. In fact $\operatorname{Tr}(A)$ is defined in terms of the usual trace of operators on $Y$. The eta invariant is defined directly in terms of $\overline{\operatorname{Tr}}$ by

$$
\eta(A)=2 \overline{\operatorname{Tr}}\left([A, t] A^{-1}\right) .
$$

Here $t$ represents the operation of multiplication in the real variable in $Y \times \mathbb{R}$. The formal trace can be obtained from the regularized trace by

$$
\widetilde{\operatorname{Tr}}(A)=i \overline{\operatorname{Tr}}([A, t]) .
$$

It is anticipated that this extended eta invariant will play a rôle in an index formula for b-pseudodifferential operators similar to that played by $\eta(\check{)})$ in the formula of Atiyah, Patodi and Singer. Indeed the possible existence of such an invariant arose first in discussion with Paolo Piazza concerning the structure of this putative formula. The results as presented in this paper are in essence a by-product of discussions with Victor Nistor concerning the K-theory of algebras of the type discussed here; the author admonishes him severely for refusing to be a co-author. The relationship between the eta homomorphism described here and K-theory will be discussed elsewhere in collaboration with Victor Nistor. The author would also like to thank Xianzhe Dai, Ezra Getzler, Rafe Mazzeo and Is Singer for their assistance.

In $\S 1$ the definition and basic properties of $\Psi_{\text {sus }}^{*}(Y ; E)$, the suspended algebra, are described. The indicial family of an element of this algebra, obtained by Fourier transformation in the real variable, is introduced in $\S 2$ and its behaviour is related to ellipticity of the element in $\S 3$. The basic regularized trace functional $\overline{\mathrm{Tr}}$ is defined in $\S 4$ and is used to define the eta functional in $\S 5$. Its identity with the eta invariant of Atiyah, Patodi and Singer for admissible Dirac operators is shown in $\S 6$; Theorem 1 above follows directly from Propositions 4 and 5. The formal trace functional is investigated in $\S 7$ and used in $\S 8$ to express the variation of the eta functional; Theorem 2 is a rephrasing of Proposition 7. This variation formula is in turn used in $\S 9$ to define the divisor flow and prove Theorem 3. 


\section{The suspended algebra}

Let $Y$ be a compact manifold without boundary. We shall consider an algebra of pseudodifferential operators on $Y \times \mathbb{R}$ which can be thought of as the suspension of the usual algebra $\Psi^{*}(Y)$ of pseudodifferential operators on $Y$.

Since $Y \times \mathbb{R}$ is a $\mathcal{C}^{\infty}$ manifold, the space $\Psi^{*}(Y \times \mathbb{R})$ of 1 -step polyhomogeneous pseudodifferential operators is well defined; it is not an algebra because the growth of the kernels is unrestricted at infinity. However the elements define operators from the space of compactly support smooth functions to smooth functions

$$
A: \mathcal{C}_{c}^{\infty}(Y \times \mathbb{R}) \longrightarrow \mathcal{C}^{\infty}(Y \times \mathbb{R})
$$

Consider the subspace of elements satisfying two conditions: translation invariance and rapid decay of the kernel.

Let $T_{\tau}: Y \times \mathbb{R} \longrightarrow Y \times \mathbb{R}$ be translation in the second variable, $T_{\tau}(y, t)=$ $(y, t-\tau)$. Thus we consider the pseudodifferential operators satisfying

$$
T_{\tau}^{*} A f=A T_{\tau}^{*} f \forall \tau \in \mathbb{R}, f \in \mathcal{C}_{c}^{\infty}(Y \times \mathbb{R}) .
$$

In terms of the Schwartz kernels of these operators this means that $A$ acts as a convolution operator in the second variable

$$
A f(y, t)=\int_{Y} \int_{\mathbb{R}} A\left(y, y^{\prime}, t-s\right) f\left(y^{\prime}, s\right) d s
$$

where $A$, as the (convolution) kernel, is a density in $y^{\prime}$. Since $A$ is a pseudodifferential operator its kernel is only singular at the submanifold $\left\{y=y^{\prime}, t=0\right\}$.

The additional constraint we place on $A$ is that its kernel lie in the space

$$
A \in \mathcal{C}_{c}^{-\infty}\left(Y^{2} \times \mathbb{R} ; \Omega Y\right)+\mathcal{S}\left(Y^{2} \times \mathbb{R} ; \Omega Y\right)
$$

where $\mathcal{S}$ denotes the Schwartz space of sections of rapid decrease, with all derivatives, at infinity.

Definition 1. For any $m \in \mathbb{R}$, let $\Psi_{\text {sus }}^{m}(Y) \subset \Psi^{m}(Y \times \mathbb{R})$ denote the space of 1-step polyhomogeneous pseudodifferential operators satisfying (1.2) and (1.4).

Note that even though the elements of $\Psi_{\text {sus }}^{m}(Y)$ are operators on $Y \times \mathbb{R}$, we think of the object itself as associated to $Y$, hence the notation.

If $E$ is a vector bundle over $Y$ the suspended space of pseudodifferential operators acting on sections of $E$ can be defined by

$$
\Psi_{\text {sus }}^{m}(Y ; E)=\Psi_{\text {sus }}^{m}(Y) \otimes_{\mathcal{C}^{\infty}(Y)} \mathcal{C}^{\infty}\left(Y^{2} ; \operatorname{Hom} E\right)
$$


where $\operatorname{Hom} E$ is the bundle over $Y^{2}$ with fibre $\operatorname{hom}\left(E_{y^{\prime}}, E_{y}\right)$ at $\left(y, y^{\prime}\right) \in Y^{2}$. The local formula (1.3) allows one to see that

$$
A \in \Psi_{\mathrm{sus}}^{m}(Y, E) \Longrightarrow A: \mathcal{S}(Y \times \mathbb{R} ; E) \longrightarrow \mathcal{S}(Y \times \mathbb{R} ; E) .
$$

Proposition 1. For any compact manifold $Y$ and bundle $E, \Psi_{\text {sus }}^{*}(Y ; E)$ is naturally a complete topological vector space and an order-filtered $*$-closed algebra.

Proof. This is a direct consequence of standard results on the composition of pseudodifferential operators. Notice that the topology is given by the best constants in the symbol estimates in local coordinates and the constants in the asymptotic expansions of the symbols in homogeneous terms, together with $\mathcal{C}^{\infty}$ estimates (and decay) on the kernels away from the 'diagonal' $\left\{y=y^{\prime}, t=0\right\}$.

\section{Indicial family}

The $*$-invariance of the algebra means, by duality, that $A \in \Psi_{\mathrm{sus}}^{m}(Y ; E)$ extends by continuity to a continuous linear map $A: \mathcal{S}^{\prime}(Y \times \mathbb{R} ; E) \longrightarrow$ $\mathcal{S}^{\prime}(Y \times \mathbb{R} ; E)$. The $t$-translation invariance, (1.2), then shows that, for any $\tau \in \mathbb{R}$ and $g \in \mathcal{C}^{\infty}(Y ; E), \widehat{A}(\tau) g=e^{-i t \tau} A\left(e^{i t \tau} g\right)$ defines a pseudodifferential operator $\widehat{A}(\tau) \in \Psi^{m}(Y ; E)$. Clearly the Schwartz kernel of $\widehat{A}(\tau)$ is obtained from that of $A$ by Fourier transformation

$$
\widehat{A}(\tau)=\int e^{-i t \tau} A\left(y, y^{\prime}, t\right) d t
$$

We call this smooth 1-parameter family of pseudodifferential operators the indicial family of $A$. By inversion of (2.1), $A$ itself can be recovered from the family $\widehat{A}(\tau)$.

\section{Ellipticity}

The elements of $\Psi_{\text {sus }}^{*}(Y ; E)$ are, by definition, 1-step polyhomogeneous (i.e., 'classical'). Thus the leading part of the locally defined symbol is a well-defined section of $\pi^{*} \operatorname{hom} E$ over $T^{*}(Y \times \mathbb{R}) \backslash 0$, where $\pi: T^{*}(Y \times$ $\mathbb{R}) \longrightarrow Y$ is the natural projection; this section is homogeneous of degree $m$. The translation invariance of the operator implies, from the uniqueness, that the symbol too is $t$-translation invariant. From (1.4) this is the only constraint on the symbol, so there is a short exact sequence

$$
\begin{aligned}
0 \longrightarrow \Psi_{\mathrm{sus}}^{m-1}(Y ; E) & \hookrightarrow \Psi_{\mathrm{sus}}^{m}(Y ; E) \\
& \stackrel{\sigma_{m}}{\longrightarrow} \mathcal{C}^{\infty}\left(S_{Y}^{*}(Y \times \mathbb{R}) ; \pi^{*} \operatorname{hom} E \otimes D_{m}\right) \longrightarrow 0
\end{aligned}
$$


Here $S^{*}(Y \times \mathbb{R})=\left(T^{*}(Y \times \mathbb{R}) \backslash 0\right) / \sim$ is the quotient by the fibre action of $\mathbb{R}^{+}, S_{Y}^{*}(Y \times \mathbb{R})$ is its restriction to $Y \times\{0\}$ and $D_{m}$ is the line bundle over $S^{*}(Y \times \mathbb{R})$ defined by the functions homogeneous of degree $m$ on $T^{*}(Y \times \mathbb{R}) \backslash 0$.

An element of $\Psi_{\mathrm{sus}}^{m}(Y ; E)$ is elliptic if $\sigma_{m}$ is invertible, as an element of the fibre of hom $E \otimes D_{m}$, with inverse in hom $E \otimes D_{-m}$, at each point. This is the same as ellipticity in the larger space $\Psi^{m}(Y \times \mathbb{R} ; E)$.

Proposition 2. If $A \in \Psi_{\text {sus }}^{m}(Y ; E)$ is elliptic then $\widehat{A}(\tau)$ is elliptic in $\Psi^{m}(Y ; E)$ for all $\tau \in \mathbb{R}$ and is invertible, with inverse in $\Psi^{-m}(Y ; E)$, for $|\tau|>C=C(A)$.

Proof. The symbol of $\widehat{A}(\tau)$ is easily computed from (2.1) in terms of the symbol of $A$. Namely, under the decomposition $T^{*}(Y \times \mathbb{R})=T^{*} Y \times \mathbb{R}_{t} \times \mathbb{R}_{\tau}$,

$$
\sigma_{m}(\widehat{A}(\tau))(y, \eta)=\sigma_{m}(y, \eta, 0)
$$

i.e., just the restriction to $\tau=0$. It follows that the symbol of $\widehat{A}(\tau)$ is independent of $\tau$ and $\widehat{A}(\tau)$ is elliptic if $A$ is.

So, assuming $A \in \Psi_{\text {sus }}^{m}(Y ; E)$ to be elliptic, standard constructions show it to have a parametrix $B \in \Psi_{\text {sus }}^{-m}(Y ; E)$. Now, the residual algebra is

$$
\Psi_{\text {sus }}^{-\infty}(Y ; E) \equiv \mathcal{S}\left(Y^{2} \times \mathbb{R} ; \Omega Y \otimes \operatorname{Hom} E\right)
$$

so the identity $A \circ B=\mathrm{Id}-R$, where $R \in \Psi_{\text {sus }}^{-\infty}(Y ; E)$ gives, on passage to indicial families, $\widehat{A}(\tau) \circ \widehat{B}(\tau)=\mathrm{Id}-\widehat{R}(\tau)$. Here $\widehat{R}(\tau) \in \mathcal{S}\left(Y^{2} \times \mathbb{R} ; \Omega Y \otimes\right.$ Hom $E)$. Thus, for $|\tau|$ large, Id $-\widehat{R}(\tau)$ is invertible with inverse $\operatorname{Id}-\widehat{S}(\tau)$, $\widehat{S}(\tau) \in \Psi^{-\infty}(Y ; E)$. It follows that $\widehat{A}(\tau)$ is invertible for $|\tau|$ large with inverse $\widehat{B}(\tau)-\widehat{B}(\tau) \cdot \widehat{S}(\tau) \in \Psi^{-m}(Y ; E)$.

It follows from (3.1) and the multiplicitivity of symbols, i.e.,

$$
\sigma_{m+m^{\prime}}(A \circ B)=\sigma_{m}(A) \cdot \sigma_{m^{\prime}}(B)
$$

that if $A$ is invertible, with inverse in $\Psi_{\text {sus }}^{-m}(Y ; E)$, then it must be elliptic. Conversely

Proposition 3. If $A \in \Psi_{\text {sus }}^{m}(Y ; E)$ is elliptic and $\widehat{A}(\tau)^{-1}$ exists, as an operator on $\mathcal{C}^{\infty}(Y ; E)$, for all $\tau \in \mathbb{R}$ then $A$ is invertible with inverse in $\Psi_{\text {sus }}^{-m}(Y ; E)$.

Proof. The invertibility of $\widehat{A}(\tau)$ implies that the inverse is $\widehat{B}(\tau)+\widehat{G}(\tau)$ with $\widehat{G} \in \mathcal{S}\left(Y^{2} \times \mathbb{R} ; \Omega Y \otimes\right.$ Hom $\left.E\right)$. Since this space is invariant under Fourier transformation it follows that the inverse of $A$ is an element of $\Psi_{\text {sus }}^{-m}(Y ; E)$. 
Note that, by Proposition 2, the invertibility of $\widehat{A}(\tau)$ is in doubt only for a bounded range of $\tau$ once it is known that $A$ is elliptic.

By summing over the translates the elements of $\Psi_{\text {sus }}^{-m}(Y ; E)$ define operators on $Y \times \mathbb{S}$ for a circle of any length; any operators acting trivially are smoothing. This relationship can be used to transfer results well known in the compact case to $\Psi_{\text {sus }}^{*}(Y ; E)$. For instance if $A \in \Psi_{\text {sus }}^{0}(Y ; E)$ then $A$ defines a bounded operator on $L^{2}(Y \times \mathbb{R} ; E)$. It also follows, as in the compact case, that the invertibility of $A$ as a bounded operator on $L^{2}(Y \times \mathbb{R} ; E)$ is equivalent to its invertibility as an element of $\Psi_{\text {sus }}^{0}(Y ; E)$.

\section{Regularized trace}

For $A \in \Psi_{\text {sus }}^{m}(Y ; E)$, we define the regularized trace by

$$
\overline{\operatorname{Tr}}(A)=\int_{Y} \operatorname{tr} A(y, y, 0), m<-\operatorname{dim} T-1 .
$$

Here $\operatorname{tr}$ is the trace functional on hom $E$. The functional $\overline{T r}$ vanishes on commutators and can be written in terms of the indicial family

$$
\overline{\operatorname{Tr}}(A)=\frac{1}{2 \pi} \int_{-\infty}^{\infty} \operatorname{Tr} \widehat{A}(\tau) d \tau .
$$

We shall use this representation to extend the functional to $\Psi_{\mathrm{sus}}^{m}(Y ; E)$ for each $m \in \mathbb{R}$.

Lemma 1. If $A \in \Psi_{\text {sus }}^{m}(Y)$ then $d^{p} \widehat{A}(\tau) / d \tau^{p} \in \Psi^{m-p}(Y ; E)$ and if $p>$ $m+\operatorname{dim} Y$ then

$$
h_{p}(\tau)=\operatorname{Tr}\left(\frac{d^{p} \widehat{A}(\tau)}{d \tau^{p}}\right) \in \mathcal{C}^{\infty}(\mathbb{R})
$$

has a complete asymptotic expansion as $\tau \rightarrow \pm \infty$ :

$$
h_{p}(\tau) \sim \sum_{l} h_{p, l}^{ \pm}|\tau|^{m-p+\operatorname{dim} Y-l} .
$$

Proof. If $A \in \Psi_{\text {sus }}^{-\infty}(Y ; E)$ then $\widehat{A} \in \mathcal{S}\left(Y^{2} \times \mathbb{R} ; \Omega Y\right)$ so for any $p, h_{p} \in \mathcal{S}(\mathbb{R})$ has a trivial asymptotic expansion. This allows us to discard any part of the kernel of $A$ outside a given neighbourhood of the diagonal of $Y^{2}$. Using a partition of unity it therefore suffices to consider $A \in \Psi_{\text {sus }}^{m}(Y ; E)$ with kernel supported in $K^{2} \times \mathbb{R}$, with $K$ a compact subset of a coordinate 
patch on $Y$. Thus we can use the local representation of pseudodifferential operators to write the kernel as

$$
A\left(y, y^{\prime}, t\right)=(2 \pi)^{-n-1} \int e^{i\left(y-y^{\prime}\right) \cdot \eta+i t \tau} a(y, \eta, \tau) d \eta d \tau\left|d y^{\prime}\right| .
$$

Here $a$ has compact support in $y$ and is a polyhomogeneous symbol of order $m$ jointly in $(\eta, \tau) \in \mathbb{R}^{n+1}, n=\operatorname{dim} Y$. This gives an explicit representation

$$
\widehat{A}(\tau)\left(y, y^{\prime}\right)=(2 \pi)^{-n} \int e^{i\left(y-y^{\prime}\right) \cdot \eta} a(y, \eta, \tau) d \eta\left|d y^{\prime}\right| .
$$

Since $a$ is a symbol, the first statement follows directly.

Furthermore, if $p>m+\operatorname{dim} Y$ then $d^{p} \widehat{A}(\tau) / d \tau^{p}$ is of trace class and

$$
h_{p}(\tau)=(2 \pi)^{-n} \iint_{\mathbb{R}^{n}} \frac{d^{p} a}{d \tau^{p}}(y, \eta, \tau) d \eta d y .
$$

The complete asymptotic expansion of $a$,

$$
a \sim \sum_{j \geq 0} a_{m-j}(y, \eta, \tau) \text { as }|(\eta, \tau)| \longrightarrow \infty
$$

where $a_{m-j}(y, \eta, \tau)$ is homogeneous of degree $m-j$, gives a complete asymptotic expansion of $h_{p}(\tau)$, as in (4.4), with

$$
h_{p, \ell}^{ \pm}=(2 \pi)^{-n} \iint \frac{d^{p} a_{m-\ell}}{d \tau^{p}}(y, \eta, \pm 1) d y d \eta
$$

The remainder terms can be estimated in the same way. Even though the proof is written for $E=\mathbb{R}$, the general case involves little more.

For $A \in \Psi_{\text {sus }}^{m}(Y ; E)$ and $h_{p}$ defined by (4.3), consider the $(p+1)$-fold integral

$$
g_{p}(\tau)=\int_{-\tau}^{\tau} \int_{0}^{\tau_{p}} \ldots \int_{0}^{\tau_{1}} h_{p}(r) d r d \tau_{1} \ldots d \tau_{p}
$$

As $\tau \rightarrow \infty$ this has a complete asymptotic expansion, as follows by integration of (4.4). Namely

$$
g_{p}(\tau) \sim \sum_{j \geq 0} g_{j} \tau^{m+1+\operatorname{dim} Y-j}+g^{\prime}(\tau)+g^{\prime \prime}(\tau) \log \tau
$$

where $g^{\prime}(\tau)$ and $g^{\prime \prime}(\tau)$ are polynomials of degree at most $p$. To prove (4.11), it is enough to integrate (4.4) term by term and hence conclude that there is a function with the same $(p+1)$-fold derivative as $g_{p}(\tau)$ with such an 
expansion. It follows that $g_{p}(\tau)$ itself can only differ from this function by a polynomial of degree $p$.

This proof actually shows that the coefficients in (4.11) depend continuously, and linearly, on the element $A$ in the topology of $\Psi_{\text {sus }}^{m}(Y ; E)$. They all define functionals on the algebra. We are interested in one in particular.

Definition 2. The (regularized) trace of $A \in \Psi_{\mathrm{sus}}^{m}(Y ; E)$ is $(2 \pi)^{-1} \times$ the coefficient of $\tau^{0}$ in the expansion (4.11) where $g_{p}(\tau)$ is defined by $(4.10)$ and (4.3) for some $p>m+\operatorname{dim} Y$.

Of course this only makes sense because of

Lemma 2. The coefficient of $\tau^{0}$ in (4.11) is independent of $p>m+\operatorname{dim} Y$ and reduces to $2 \pi \overline{\operatorname{Tr}}(A)$ defined by (4.2) if $m<-\operatorname{dim} Y-1$.

Proof. It suffices to consider the effect of the increase of $p$. Since

$$
h_{p+k}(\tau)=\left(\frac{d}{d \tau}\right)^{k} h_{p}(\tau)
$$

for $k \geq 0$ and $p \geq m+\operatorname{dim} Y$, the effect of the extra $k$ integrals on defining $g_{p+k}$ is that

$$
g_{p+k}(\tau)=\int_{-\tau}^{\tau} \int_{0}^{\tau_{p}} \ldots \int_{0}^{\tau_{1}}\left(h_{p}(r)+g_{k}(r)\right) d r d \tau_{1} \ldots d \tau_{p}
$$

where $g_{k}(r)$ is a polynomial of degree $k-1$. The next $p$ integrals give a polynomial of degree $p+k-1$ and the last integral over $[-\tau, \tau]$ gives a polynomial without constant term. Thus the coefficient of $\tau^{0}$ in $g_{p+k}(\tau)$ as $\tau \rightarrow \infty$ is the same as that in $g_{p}(\tau)$. This shows that $\overline{\operatorname{Tr}}(A)$ is well defined and reduces to (4.2) if $m<-\operatorname{dim} Y-1$.

Lemma 3. The functional $\overline{\operatorname{Tr}}$ vanishes on commutators.

Thus the functional $\overline{\mathrm{Tr}}$ is invariant under conjugation in the algebra.

Proof. If $A, B \in \Psi_{\text {sus }}^{*}(Y ; E)$ then the indicial family of the commutator is

$$
\widehat{[A, B]}(\tau)=[\widehat{A}(\tau), \widehat{B}(\tau)]
$$

Moreover

$$
\frac{d}{d \tau}[\widehat{A}(\tau), \widehat{B}(\tau)]=\left[\frac{d \widehat{A}(\tau)}{d \tau}, \widehat{B}(\tau)\right]+\left[\widehat{A}(\tau), \frac{d \widehat{B}(\tau)}{d \tau}\right]
$$

is a sum of commutators; each term in the sum has the sum of orders reduced by 1 . Proceeding iteratively $\left(\frac{d}{d \tau}\right)^{p}[\widehat{A}(\tau), \widehat{B}(\tau)]$ is therefore a sum 
of commutators where each term has total order ord $(A)+\operatorname{ord}(B)-p$. Choosing $p$ large enough it follows that the trace of such a commutator vanishes. Thus $h_{p}(\tau) \equiv 0$ for $p$ large, so $\overline{\operatorname{Tr}}([A, B])=0$.

Notice that $\overline{\operatorname{Tr}}(A)=0$ if the kernel of $A$ is odd under the reflection $t \mapsto-t$, or equivalently if $\widehat{A}(-\tau)=-\widehat{A}(\tau)$.

\section{Eta invariant}

If $A \in \Psi_{\mathrm{sus}}^{m}(Y ; E)$ then the commutator $[A, t] \in \Psi_{\mathrm{sus}}^{m-1}(Y ; E)$ since it has Schwartz kernel $-A\left(y, y^{\prime}, t\right) t$.

Definition 3. If $A \in \Psi_{\mathrm{sus}}^{m}(Y ; E)$ is elliptic and invertible, the eta invariant of $A$ is defined to be

$$
\eta(A)=2 \overline{\operatorname{Tr}}\left([A, t] A^{-1}\right) .
$$

Proposition 4. The eta invariant is an additive homomorphism from the group of invertible elements of $\Psi_{\text {sus }}^{*}(Y ; E)$ into $\mathbb{C}$.

Proof. If $A, B \in \Psi_{\text {sus }}^{*}(Y ; E)$ then $[A B, t]=[A, t] B+A[B, t]$. If both are invertible then $[A B, t](A B)^{-1}=[A, t] A^{-1}+A[B, t] B^{-1} A^{-1}$. The conjugation invariance of $\overline{\mathrm{Tr}}$ then shows that

$$
\eta(A B)=\eta(A)+\eta(B) .
$$

Observe that the indicial family of $[A, t]$ is $\frac{1}{i} \partial \widehat{A}(\tau) / \partial \tau$. Using (4.2) this gives

Lemma 4. If $A=\mathrm{Id}+S$ with $S \in \Psi_{\mathrm{sus}}^{m}(Y ; E), m<-\operatorname{dim} Y$ such that $A$ is invertible then

$$
\eta(A)=\frac{1}{\pi i} \int_{-\infty}^{\infty} \operatorname{Tr}\left(\frac{\partial \widehat{S}(\tau)}{\partial \tau}(\operatorname{Id}+\widehat{S}(\tau))^{-1}\right) d \tau .
$$

Since $\widehat{S}(\tau)$ is trace class, $\operatorname{det}(\operatorname{Id}+\widehat{S}(\tau))$ is well defined and (5.3) shows that $\eta(A)$ is the variation of the argument of $\operatorname{det}(\operatorname{Id}+\widehat{S}(\tau))$ as $\tau$ ranges from $-\infty$ to $\infty$. It is in other words the winding number of the map $(-\infty, \infty) \ni \tau \longmapsto \operatorname{det}(\operatorname{Id}+\widehat{S}(\tau)) \in \mathbb{C}$ which has end points 1 and never takes the value 0 . In the general case, $\eta(A)$ is by definition $(\pi i)^{-1} \times$ the coefficient of $\tau^{0}$ as $\tau \rightarrow \infty$ in the expansion of

$$
\begin{gathered}
\int_{-\tau}^{\tau} \int_{0}^{\tau_{p}} \ldots \int_{0}^{\tau_{1}} \operatorname{Tr}\left(\frac{d}{d \tau}\right)^{p}\left(\frac{\partial \widehat{A}(s)}{\partial \tau} \widehat{A}(s)^{-1}\right) d s d \tau_{1} \ldots d \tau_{p} \\
\text { for any } p>\operatorname{dim} Y .
\end{gathered}
$$




\section{Dirac operators}

Let $E$ be an Hermitian Clifford module for a metric on $Y$, which has odd dimension, with a unitary Clifford connection specified. Let $\partial$ be the associated Dirac operator on sections of $E$; we shall describe such a Dirac operator as admissible. The differential operators

$$
\check{\partial}_{\mathrm{sus}}^{ \pm}=\check{\partial} \pm i D_{t} \in \Psi_{\mathrm{sus}}^{1}(Y ; E), D_{t}=\frac{1}{i} \frac{\partial}{\partial t},
$$

are elliptic. Since $\partial$ is self-adjoint and $\widehat{\partial}_{\text {sus }}^{ \pm}=\partial \partial \pm i \tau$, it follows from Proposition 3 that $\partial_{\text {sus }}^{ \pm}$are invertible with inverses in $\Psi_{\text {sus }}^{-1}(Y ; E)$ if and only if $\partial$ is invertible.

Proposition 5. For any invertible admissible Dirac operator

$$
\eta\left(\widetilde{\partial}_{\text {sus }}^{ \pm}\right)= \pm \frac{1}{\sqrt{\pi}} \int_{0}^{\infty} t^{-\frac{1}{2}} \operatorname{Tr}\left(ð e^{-t \varpi^{2}}\right) d t= \pm \eta(ð)
$$

is \pm the eta invariant of $\partial$ as defined by Atiyah, Patodi and Singer.

Proof. Formally if $1=(\sqrt{\pi})^{-1} \int_{-\infty}^{\infty} t^{\frac{1}{2}} \exp \left(-t \tau^{2}\right) d \tau$ is inserted into the integral in (6.2) and the $t$ integral is carried out first, the result is

$$
(\pi)^{-1} \int_{-\infty}^{\infty} \operatorname{Tr} ð\left(ð^{2}+\tau^{2}\right)^{-1} d \tau
$$

which reduces to $\eta\left(\check{\partial}^{+}\right)$. To justify this exchange of order of integration, the special properties of admissible Dirac operators, in particular the local index theorem, will be used. This means that the function

$$
h(t)=\operatorname{Tr}\left(ð e^{-t \widetilde{\partial}^{2}}\right) \in t^{\frac{1}{2}} \mathcal{C}^{\infty}([0, \infty)) .
$$

It is also exponentially decreasing as $t \rightarrow \infty$.

Consider the integral

$$
g(s, \tau)=\int_{s}^{\infty} e^{-t \tau^{2}} h(t) d t, s \geq 0 .
$$

This is a $\mathcal{C}^{\infty}$ function of $s^{\frac{1}{2}}$ in $s \geq 0, \tau \in \mathbb{R}$. Moreover, as $\tau \rightarrow \infty$, it is rapidly decreasing if $s>0$ and the smoothness and vanishing in (6.3) gives uniform bounds

$$
\left|\left(\tau \partial_{\tau}\right)^{p} g(s, \tau)\right| \leq C_{p}\left(1+\tau^{2}\right)^{-1}, s \geq 0, \tau \in \mathbb{R} .
$$


Thus, $g$ is uniformly a symbol of order -2 as $s \downarrow 0$. The order of the limits in the following expression can be exchanged to show that

$$
\eta(ð)=\frac{1}{\sqrt{\pi}} \int_{0}^{\infty} t^{-\frac{1}{2}} \operatorname{Tr}\left(ð e^{-t \check{\partial}^{2}}\right) d t=\frac{1}{\pi} \lim _{s \downarrow 0} \int_{-\infty}^{\infty} g(s, \tau) d \tau .
$$

Since we wish to arrive at $(4.10)$, set $g_{p}^{\prime}(s, \tau)=g(s, \tau)-\sum_{j<p} \frac{\tau^{j}}{j !} \partial_{\tau}^{j} g(s, 0)$ and observe that $g_{p}(s, \tau)=\int_{-\tau}^{\tau} g_{p}^{\prime}(s, r) d r$ has a uniform asymptotic expansion as $\tau \rightarrow \infty$, as $s \downarrow 0$, down to terms of order $|\tau|^{-1}$; i.e., with error uniformly bounded by a multiple of $|\tau|^{-2}$. Thus, from (6.6)

$$
\begin{aligned}
& \eta(\check{\partial})=\frac{1}{\pi} \lim _{s \downarrow} \int_{-\infty}^{\infty} g(s, \tau) d \tau \\
& =\frac{1}{\pi} \times \text { the coefficient of } \tau^{0} \text { in the expansion of } g_{p}(0, \tau) \text {. }
\end{aligned}
$$

On the other hand, for $s=0$, this corresponds to the even part of the function in (4.10) for $A$ with indicial family

$$
\widehat{A}(\tau)=\int_{0}^{\infty} e^{-t \tau^{2}}( \pm i \tau+\partial) e^{-t \widetilde{ठ}^{2}} d t=(\mp i \tau+ð)^{-1} .
$$

Since the odd part does not contribute to $\overline{T r}$ and $\frac{\partial}{\partial \tau}( \pm i \tau+\partial)= \pm i$, we finally conclude that

$$
\eta(ð)= \pm \eta\left(\mathrm{\partial}_{\text {sus }}^{ \pm}\right)= \pm \overline{\operatorname{Tr}}\left(\left[\varlimsup_{\text {sus }}^{ \pm}, t\right]\left(\mathrm{\partial}_{\text {sus }}^{ \pm}\right)^{-1}\right) .
$$

\section{Formal trace}

To capture the variation of the eta functional we define a second 'trace' functional on the suspended algebra. If $A \in \Psi_{\text {sus }}^{m}(Y ; E)$ consider the analogue of (4.10) but with one less integral

$$
\tilde{g}_{p}(\tau)=\int_{-\tau}^{\tau} \int_{0}^{\tau_{p-1}} \ldots \int_{0}^{\tau_{1}} h_{p}(r) d r d \tau_{1} \ldots d \tau_{p-1},
$$

where $h_{p}$ is still given by (4.3).

Definition 4. If $A \in \Psi_{\text {sus }}^{m}(Y ; E)$ the formal trace, $\widetilde{\operatorname{Tr}}(A)$, is defined to be $(2 \pi)^{-1} \times$ the coefficient of $\tau^{0}$ in the expansion of $\tilde{g}_{p}(\tau)$ as $\tau \rightarrow \infty$. As for $\overline{\operatorname{Tr}}(A), \widetilde{\operatorname{Tr}}(A)$ is independent of the choice of $p$, provided $p>m+\operatorname{dim} Y$. 
Lemma 5. If $A \in \Psi_{\text {sus }}^{m}(Y ; E)$ with $m<-\operatorname{dim} Y$ then $\widetilde{\operatorname{Tr}}(A)=0$.

Proof. If the order of $A$ is less than $-\operatorname{dim} Y$ then $\tilde{h}_{p}(\tau)$ can have no constant term in its asymptotic expansion.

Lemma 6. For any $A \in \Psi_{\mathrm{sus}}^{m}(Y ; E)$

$$
\widetilde{\operatorname{Tr}}(A)=i \overline{\operatorname{Tr}}([A, t]) \text {. }
$$

Proof. Since $\widehat{[A, t]}(\tau)=\frac{1}{i} \frac{\partial}{\partial \tau} \widehat{A}(\tau)$, this is by definition.

In particular this shows that $\widetilde{\operatorname{Tr}}([A, B])=0$ if $A, B \in \Psi_{\text {sus }}^{*}(Y ; E)$ since by Jacobi's identity $[[A, B], t]=[A,[B, t]]+[[A, t], B]$ is a sum of commutators in the algebra.

As for the closely related 'residue trace' of Guillemin and Wodzicki, it is straightforward to give an explicit formula for the formal trace in terms of the symbol expansion for the operator. Since $\widetilde{T r}$ is linear and vanishes on low order operators, it suffices to consider $A$ of the form (4.5) with amplitude $a(y, \eta, \tau)$ compactly supported, in $y$, in a coordinate patch over which $E$ is trivial.

Proposition 6. If $A \in \Psi_{\mathrm{sus}}^{m}(Y ; E)$ is of the form (4.5) with support in a coordinate patch over which $E$ is trivial then $\widetilde{\operatorname{Tr}}(A)=0$ if $m \notin \mathbb{Z}$ or $m<-\operatorname{dim} Y$ and

$$
\begin{gathered}
\widetilde{\operatorname{Tr}}(A)=\lim _{L \rightarrow \infty} \iint_{\{|\eta| \leq L\}}\left(\operatorname{tr} a_{m-q}(y, \eta, 1)-\operatorname{tr} a_{m-q}(y, \eta,-1)\right) \omega^{n}, \\
n=q-m=\operatorname{dim} Y,
\end{gathered}
$$

where $\omega$ is the symplectic form on $T^{*} Y$ and $\operatorname{tr}$ is the trace functional on hom $E$.

Proof. It is enough to consider the case that $E=\mathbb{R}$. Using (4.5) and (4.7) the formula (7.1) becomes

$$
\tilde{g}_{p}(\tau)=\int_{-\tau}^{\tau} \int_{0}^{\tau_{p-1}} \ldots \int_{0}^{\tau_{1}} \int_{\mathbb{R}^{2 n}} \frac{d^{p}}{d s^{p}} a(y, \eta, s) d y d \eta d s d \tau_{1} \ldots d \tau_{p-1} .
$$

Let $\varphi \in \mathcal{C}^{\infty}\left(\mathbb{R}^{n+1}\right)$ have $\varphi(\eta, \tau)=1$ in $|(\eta, \tau)| \geq 1$ and $\varphi(\eta, \tau)=0$ in $|(\eta, \tau)| \leq \frac{1}{2}$. Then $a$ in (7.4) can be replaced by the finite sum

$$
a \longmapsto \sum_{m-j \geq-\operatorname{dim} Y} \varphi(\eta, \tau) a_{m-j}(y, \eta, \tau)
$$


Here the $a_{m-j}$ are homogeneous of degree $m-j$, and homogeneous terms of degree less than $-\operatorname{dim} Y$ can be dropped because of Lemma 5 . It therefore suffices to consider (7.4) with one such term.

Now $a_{m-j}$, which we will denote by $b$, is homogeneous of degree greater than or equal to $-\operatorname{dim} Y$. It therefore defines a locally integrable function, and hence a distribution, on $\mathbb{R}^{n} \times \mathbb{R}^{n} \times \mathbb{R}$; this will be denoted $\mu(y, \eta, \tau)=$ $b(y, \eta, \tau) \in \mathcal{C}^{-\infty}\left(\mathbb{R}^{2 n+1}\right)$. The difference $\mu-\varphi b \in \mathcal{C}^{-\infty}\left(\mathbb{R} \times \mathbb{R}^{n+1}\right)$ has compact support. We know that $\widetilde{\operatorname{Tr}}(A)$ is not affected by a polynomial term in $\tilde{h}_{p}(\tau)$ so the contribution of this term to $\tilde{g}_{p}(\tau)$ is asymptotically zero.

Thus, $\widetilde{\operatorname{Tr}}(A)$ is the coefficient of $\tau^{0}$ as $\tau \rightarrow \infty$ in

$$
\int_{-\tau}^{\tau} \int_{-1}^{\tau_{p-1}} \cdots \int_{-1}^{\tau_{1}} \int_{\mathbb{R}^{2 n}} \frac{d^{p}}{d s^{p}} \mu(y, \eta, s) d y d \eta d s d \tau_{1} \ldots d \tau_{p-1}
$$

Here the origin of integration has been moved to a point near which the integrand is smooth. Now the integral over $\mathbb{R}^{2 n}$ is a distribution in $s$ which is homogeneous of degree $\operatorname{deg} b+\operatorname{dim} Y-p$. The $p$ integrals therefore give a distribution homogeneous of $\operatorname{degree} \operatorname{deg} b+\operatorname{dim} Y$ plus a polynomial. Taking the even part gives no constant term at infinity unless $\operatorname{deg} b=$ $-\operatorname{dim} Y$.

In the remaining case, $\operatorname{deg} b=-\operatorname{dim} Y ; p$ in (7.6) can be taken as 1 . Thus we need to compute $\int_{-\tau}^{\tau} \int_{\mathbb{R}^{2 n}} \frac{d}{d s} \mu(y, \eta, s) d y d \eta d s$ for $\tau>0$ since this must be constant. This is the volume integral of the form

$$
d(\mu(y, \eta, \tau) d y d \eta)=\frac{d}{d \tau} \mu(y, \eta, \tau) d \tau d y d \eta
$$

By Stokes' theorem

$$
\int_{-1}^{1} \int_{\mathbb{R}^{2 n}} \frac{\partial \mu}{\partial \tau} d \tau d y d \eta=\int_{|\eta| \leq L} b(y, \eta, 1) d s d \eta-\int_{|\eta| \leq L} b(y, \eta,-1) d y d \eta
$$

The terms on the left do not converge, separately, as $L \rightarrow \infty$. Indeed they will in general diverge logarithmically but the coefficients of $\log L$ will cancel. 


\section{Variation of eta}

The smooth dependence of $\overline{\operatorname{Tr}}(A)$ on $A$ shows that if $A_{s}$ is a 1-parameter family in $\Psi_{\text {sus }}^{m}(Y ; E)$ which depends smoothly on $s \in(-\epsilon, \epsilon)$ and is elliptic and invertible then $\eta\left(A_{s}\right)$ is smooth.

Proposition 7. If $A_{s} \in \Psi_{\mathrm{sus}}^{m}(Y ; E)$ is elliptic and invertible and depends smoothly on $s \in(-\epsilon, \epsilon)$ then

$$
\frac{d}{d s} \eta\left(A_{s}\right)=\frac{2}{i} \widetilde{\operatorname{Tr}}\left(\frac{d A_{s}}{d s} A_{s}^{-1}\right) .
$$

Proof. From the definition, (5.1), of $\eta$

$$
\frac{d}{d s} \eta\left(A_{s}\right)=2 \overline{\operatorname{Tr}}\left(\left[\frac{d A_{s}}{d s}, t\right] A_{s}^{-1}-\left[A_{s}, t\right] A_{s}^{-1} \frac{d A_{s}}{d s} A_{s}^{-1}\right)
$$

where the usual identity for the variation of the inverse has been used. Moreover

$$
0=\left[A_{s} A_{s}^{-1}, t\right]=A_{s}\left[A_{s}^{-1}, t\right]+\left[A_{s}, t\right] A_{s}^{-1}
$$

so from (8.2) and the conjugation invariance of $\overline{\mathrm{Tr}}$ it follows that

$$
\frac{d}{d s} \eta\left(A_{s}\right)=2 \overline{\operatorname{Tr}}\left(\left[\frac{d A_{s}}{d s}, t\right] A_{s}^{-1}+\left[A_{s}^{-1}, t\right] \frac{d A_{s}}{d s}\right) .
$$

Thus

$$
\frac{d}{d s} \eta\left(A_{s}\right)=2 \overline{\operatorname{Tr}}\left(\left[\frac{d A_{s}}{d s} A_{s}^{-1}, t\right]\right)=\frac{2}{i} \widetilde{\operatorname{Tr}}\left(\frac{d A_{s}}{d s} A_{s}^{-1}\right)
$$

by Lemma 6 .

Thus, just as for the eta invariant in the usual sense, the variation is 'local'. We can exploit this by noting that if $B_{s} \in \Psi_{\text {sus }}^{m}(Y ; E)$ is a smooth family of elliptic, but not necessarily invertible, operators then the 'variation of the eta function'

$$
v \eta\left(B_{s}\right)=\frac{2}{i} \widetilde{\operatorname{Tr}}\left(\frac{d B_{s}}{d s} Q_{s}\right), B_{s} \circ Q_{s} \equiv \mathrm{Id} \bmod \Psi_{\text {sus }}^{-\infty}(Y ; E)
$$

is defined independent of the choice of parametrix $Q_{s} \in \Psi_{\text {sus }}^{-m}(Y ; E)$. It is smooth in $s$, since $Q_{s}$ can be chosen to be smooth. 


\section{Divisor flow}

Let $\operatorname{Ell}_{m} \subset \Psi_{\text {sus }}^{m}(Y ; E)$ be the open set of elliptic elements and let $\operatorname{Ell}_{m}(A)$, for $A \in \operatorname{Ell}_{m}$, denote the component containing $A$.

Definition 5. If $A \in \Psi_{\text {sus }}^{m}(Y ; E)$ is elliptic and invertible and $B \in \operatorname{Ell}_{m}(A)$ is invertible, the divisor flow from $A$ to $B$ is

$$
D F(B, A)=\frac{1}{2}\left(\eta(B)-\eta(A)-\int_{0}^{1} v \eta\left(B_{s}\right) d s\right)
$$

where $B_{s}$ is a smooth family in $\operatorname{Ell}_{m}(A)$ with $B_{0}=A, B_{1}=B$.

Clearly the divisor flow from $B$ to $A$ does not depend on the path chosen. Indeed, by Proposition 7 , it is constant on the components of $\operatorname{Inv}_{m} \cap \operatorname{Ell}_{m}(A)$, where $\operatorname{Inv}_{m} \subset \Psi_{\text {sus }}^{m}(Y ; E)$ is the open set of invertible elliptic elements.

Theorem 4. For each $A \in \Psi_{\text {sus }}^{m}(Y ; E)$ elliptic and invertible, $\mathrm{DF}_{A}(B)=$ $\mathrm{DF}(B, A)$ defines a surjective map

$$
\mathrm{DF}_{A}: \operatorname{Inv}_{m} \cap \operatorname{Ell}_{m}(A) \longrightarrow \mathbb{Z}
$$

which parametrizes the components, i.e., if $\mathrm{DF}_{A}(B)=\mathrm{DF}_{A}\left(B^{\prime}\right)$ with $B, B^{\prime} \in \operatorname{Ell}_{m}(A) \cap \operatorname{Inv}_{m}$ then $B$ and $B^{\prime}$ are connected by a smooth curve of elliptic and invertible operators.

Proof. It is enough to prove this result for one fixed invertible elliptic operator $A \in \Psi_{\text {sus }}(Y ; E)$ since if $G$ is another such operator of order $m^{\prime}$ and $B \in \operatorname{Ell}_{m^{\prime}}(G) \cap \operatorname{Inv}_{m^{\prime}}$ then $B^{\prime}=B G^{-1} A \in \operatorname{Ell}_{m}(G) \cap \operatorname{Inv}_{m}$ and

$$
\begin{aligned}
\mathrm{DF}_{G}(B) & =\frac{1}{2}\left(\eta(B)-\eta(G)-\int_{0}^{1} \mathrm{v} \eta\left(Q_{s}\right) d s\right) \\
& =\frac{1}{2}\left(\eta\left(B^{\prime}\right)-\eta(A)-\int_{0}^{1} \mathrm{v} \eta\left(Q_{s}^{\prime}\right) d s\right) \\
& =\mathrm{DF}_{A}\left(B^{\prime}\right)=\mathrm{DF}_{A}\left(B G^{-1} A\right)
\end{aligned}
$$

where $Q_{s}$ is a smooth curve in $\operatorname{Ell}_{m^{\prime}}(G)$ connecting $B$ and $G$ so $Q_{s}^{\prime}=$ $Q_{s} G^{-1} A$ is a smooth curve in $\operatorname{Ell}_{m}(A)$ connecting $B^{\prime}$ and $A$. Acting by composition on the right, $G^{-1} A$ maps the components of $\operatorname{Ell}_{m^{\prime}}(G) \cap \operatorname{Inv}_{m^{\prime}}$ to those of $\operatorname{Ell}_{m}(A) \cap \operatorname{Inv}_{m}$. For simplicity we shall therefore take $A=\operatorname{Id}$.

We proceed to a more special version of the desired result from which we then deduce the general case. 
Proposition 8. On the algebra of operators $\mathcal{A}=\operatorname{Id}+\Psi_{\text {sus }}^{-\infty}(Y ; E)$, the function $\mathrm{DF}_{\mathrm{Id}}$ maps onto the integers, is constant on the components of $\mathcal{A} \cap \operatorname{Inv}_{0}$, satisfies

$$
\mathrm{DF}_{\mathrm{Id}}\left((\operatorname{Id}+S)\left(\operatorname{Id}+S^{\prime}\right)\right)=\mathrm{DF}_{\mathrm{Id}}(\operatorname{Id}+S)+\mathrm{DF}_{\mathrm{Id}}\left(\operatorname{Id}+S^{\prime}\right),
$$

and $\mathrm{DF}_{\mathrm{Id}}(\mathrm{Id}+S)=\mathrm{DF}_{\mathrm{Id}}\left(\mathrm{Id}+S^{\prime}\right)$ for $S, S^{\prime} \in \Psi_{\text {sus }}^{-\infty}(Y ; E)$ implies that $\mathrm{Id}+S$ and $\mathrm{Id}+S^{\prime}$ are in the same component of $\mathcal{A} \cap \mathrm{Inv}_{0}$; in particular $\mathrm{DF}(\cdot, \cdot)$ is a 0 -cycle on $\mathcal{A}$ spanning $H^{0}(\mathcal{A} ; \mathbb{Z})$.

Proof. Certainly $\mathrm{DF}_{\mathrm{Id}}(\mathrm{Id}+S)$ is constant on the components of $\mathcal{A} \cap \operatorname{Inv}_{0}$ since its local variation vanishes. Choose an Hermitian connection on the bundle $E$ and consider the connection Laplacian $\Delta^{\nabla}$. This is an elliptic, nonnegative, self-adjoint operator. Let $P_{j}$ be the projection onto the span of the eigenfunctions of $\Delta^{\nabla}$ with eigenvalue less than $j$. Let $S_{j}$ be the kernel of the operator $P_{j} \circ S \circ P_{j}$. This sequence converges in $\mathcal{S}\left(Y^{2} \times \mathbb{R} ; \Omega \otimes \operatorname{Hom} E\right)$ to the kernel of $S$ as $j \rightarrow \infty$. Moreover, for large enough $j,\left\|S-S_{j}\right\|<\frac{1}{2}$ acting on $L^{2}(Y \times \mathbb{R} ; E)$ so that for $r \in[0,1]$, Id $+r S+(1-r) S_{j}$ is invertible on $L^{2}(Y \times \mathbb{R} ; E)$ and hence as an element of $\mathcal{A}$. Now

$$
\begin{aligned}
\mathrm{DF}_{\mathrm{Id}}\left(\mathrm{Id}+S_{j}\right) & =\frac{1}{2 \pi i} \int_{-\infty}^{\infty} \operatorname{Tr}\left(\frac{d \widehat{S}_{j}(\tau)}{d \tau}\left(\operatorname{Id}+\widehat{S}_{j}(\tau)\right)^{-1}\right) d \tau \\
& =\frac{1}{2 \pi i} \int_{-\infty}^{\infty} \frac{d}{d \tau} \log \operatorname{det}\left(\mathrm{Id}+\widehat{S}_{j}(\tau) d \tau\right.
\end{aligned}
$$

is the winding number of the curve $\operatorname{Id}+\widehat{S}_{j}(\tau)$ considered as a curve into $\mathrm{GL}(N ; \mathbb{C})$ where $N$ is the rank of $P_{j}$. It is therefore an integer and (9.4) follows. Moreover this function captures $\pi_{1}(\mathrm{GL}(N ; \mathbb{C}))$.

Thus if $S^{\prime}$ is another such operator with $\mathrm{Id}+S^{\prime}$ invertible then, taking $j$ large enough, the equality $\mathrm{DF}_{\mathrm{Id}}(\mathrm{Id}+S)=\mathrm{DF}_{\mathrm{Id}}\left(\mathrm{Id}+S^{\prime}\right)$ implies that the curves $\operatorname{Id}+\widehat{S}_{j}(\tau)$ and $\operatorname{Id}+\widehat{S}_{j}^{\prime}(\tau)$ are homotopic as curves in $\operatorname{GL}(N ; \mathbb{C})$. This homotopy can certainly be arranged to be linear in the parameter for $|\tau|$ large and hence to be the indicial family of an homotopy in $\mathcal{A}$. Since the surjectivity of $\mathrm{DF}_{\mathrm{Id}}$ follows similarly, this completes the proof of the proposition.

Returning to the proof of Theorem 4 we first show that if $A \in \operatorname{Ell}_{0}$ (Id) $\cap$ $\operatorname{Inv}_{0}$ then $\operatorname{DF}_{\operatorname{Id}}(A) \in \mathbb{Z}$. Let $A_{s} \in \operatorname{Ell}_{0}(\mathrm{Id})$ be a curve with $A_{0}=\mathrm{Id}$ and $A_{1}=A$. Choosing $\rho \in \mathcal{C}_{c}^{\infty}(\mathbb{R})$ with $\rho(t)=1$ for $|t|<1$ and $\rho(t)=$ 0 for $|t|>2$ consider the family with kernels $A_{s}\left(y, y^{\prime}, t\right) \rho(t / n)$. For $n$ sufficiently large, $A \rho(t / n)$ must still be invertible since $(1-\rho(t / n)) A \longrightarrow 0$ in $\mathcal{S}\left(Y^{2} \times \mathbb{R} ; \Omega Y \otimes \operatorname{Hom} E\right)$. Thus we can assume that all the $A_{s}$ have (uniformly) compactly supported convolution kernels. 
Lemma 7. For $A \in \Psi_{\text {sus }}^{m}(Y ; E)$ elliptic, with compactly supported (convolution) kernel, the indicial family $\widehat{A}(\tau)$ is entire with values in $\Psi^{m}(Y ; E)$ and with divisor (set of points of non-invertibility) a discrete set contained in a region such that $|\operatorname{Re} \tau| \rightarrow \infty$ as $|\tau| \rightarrow \infty$.

Proof. The ellipticity of $A$ allows a parametrix $B \in \Psi_{\text {sus }}^{-m}(Y ; E)$ to be constructed and $B$ can also be taken to have compactly supported kernel. The error term, $R=A \circ B-\mathrm{Id} \in \Psi_{\text {sus }}^{\infty}(Y ; E)$, therefore has kernel in $\mathcal{C}_{c}^{\infty}\left(Y^{2} \times \mathbb{R} ; \Omega Y \otimes \operatorname{Hom} E\right)$. The indicial families are all entire, as the Fourier(-Laplace) transforms of compactly supported distributions. The indicial family of the error term satisfies

$$
|\widehat{R}(\tau)| \leq C_{N}(|\operatorname{Im} \tau|)(1+|\tau|)^{-N}
$$

for each $N$, with $C_{N}(r)$ some function. This proves the invertibility outside a set as indicated and the discreteness of the divisor follows from analytic Fredholm theory.

For any $r \in \mathbb{R}$ the family $\mathbb{R} \ni \tau \longrightarrow \widehat{A}(\tau+i r)$ is the indicial family of $A\left(y, y^{\prime}, t\right) \exp (r t)$. By Lemma 7 the set of points $r \in \mathbb{R}$ such that $\widehat{A}(\tau+i r)$ is not invertible for all $\tau \in \mathbb{R}$ is discrete in $\mathbb{R}$. Thus for each $s \in[0,1]$ there is certainly some (small) value of $r$ such that $A_{s} \exp (r t) \in \Psi_{\mathrm{sus}}^{0}(Y ; E)$ is invertible. The estimates in (9.6) are uniform in $s \in[0,1]$ so $A_{s} \exp (r t)$ must remain invertible for nearby $s$. Thus we can cover $[0,1]$ by a finite number of closed intervals $\left[s_{j-1}, s_{j}\right]$ with corresponding constants $r_{j} \in \mathbb{R}$ such that for $s \in\left[s_{j-1}, s_{j}\right]$ the operator with kernel $A_{s, j}^{\prime}=A_{s} \exp \left(r_{j} t\right)$ is invertible. Now $\operatorname{DF}_{\operatorname{Id}}\left(A_{s, j}^{\prime}\right)$ must be constant on these intervals, so it suffices to show that the difference $\operatorname{DF}_{\mathrm{Id}}\left(A_{s_{j}, j}\right)-\mathrm{DF}_{\mathrm{Id}}\left(A_{s_{j}, j+1}\right)$ is an integer for each $j$.

The difference of operators

$$
Q=A_{s_{j}, j}-A_{s_{j}, j+1}=A_{s}\left(y, y^{\prime}, t\right)\left(\exp \left(r_{j} t\right)-\exp \left(r_{j+1} t\right)\right) \in \Psi_{\text {sus }}^{-1}(Y ; E)
$$

is of order -1 , since the symbol of $A_{s} \exp (r t)$ is independent of $r$. It follows that $Q$ can be norm approximated by operators in $\Psi_{\text {sus }}^{-\infty}(Y ; E)$, this can be done in the topology of pseudodifferential operators of order $-1 / 2$ 'with bounds' (but not in the polyhomogeneous topology). If $Q^{\prime}$ is a sufficiently close approximation then

$$
M_{u}=A_{s_{j}, j+1}+u\left(Q-Q^{\prime}\right)=A_{s_{j}, j}-Q+u\left(Q-Q^{\prime}\right)
$$

must be invertible for $u \in[0,1]$. Thus $\operatorname{DF}_{\mathrm{Id}}\left(A_{s_{j}, j+1}\right)=\operatorname{DF}_{\mathrm{Id}}\left(A_{s_{j}, j}-Q^{\prime}\right)$. So we are reduced to showing that

$$
\operatorname{DF}_{\mathrm{Id}}\left(A_{s_{j}, j}\right)-\operatorname{DF}_{\mathrm{Id}}\left(A_{s_{j}, j}-Q^{\prime}\right) \in \mathbb{Z}
$$


Now set $\left(A_{s_{j}, j}-Q^{\prime}\right) A_{s_{j}, j}^{-1}=\operatorname{Id}-Q^{\prime \prime}$, so $Q^{\prime \prime} \in \Psi_{\text {sus }}^{-\infty}(Y ; E)$ and

$$
\begin{aligned}
\operatorname{DF}_{\mathrm{Id}}\left(A_{s_{j}, j}\right)-\mathrm{DF}_{\mathrm{Id}}\left(A_{s_{j}, j}-Q^{\prime}\right) & =-\mathrm{DF}\left(A_{s_{j}, j}^{\prime}, A_{s_{j}, j}-Q^{\prime}\right) \\
& =\mathrm{DF}_{\mathrm{Id}}\left(\mathrm{Id}-Q^{\prime \prime}\right) \in \mathbb{Z}
\end{aligned}
$$

by (9.3) and Proposition 8. Thus, using (9.3), $\mathrm{DF}_{A}(B) \in \mathbb{Z}$ whenever $A, B$ are elliptic and invertible and $B \in \operatorname{Ell}_{m}(A)$.

Now we suppose that $A \in \operatorname{Ell}_{0}(\mathrm{Id}) \cap \operatorname{Inv}_{0}$ has $\operatorname{DF}_{\operatorname{Id}}(A)=0$; we wish to show that it can be connected to Id by a curve in the invertible set $\operatorname{Inv}_{0} \cap \operatorname{Ell}_{0}(\mathrm{Id})$. Consider again the curve linking $A$ to Id in $\mathrm{Ell}_{0}(\mathrm{Id})$. Inserting the linear homotopies as in (9.8) to modify the $A_{s_{j}, j+1}$ to be $A_{s_{j}, j}-Q_{j}$ with $Q_{j} \in \Psi_{\text {sus }}^{-\infty}(Y ; E)$ and renormalizing the parameter, renamed to $r$, the curve can be assumed to consist of $2 p-1$ segments of parameter length one alternating between $p$ seqments on which the elements are invertible and $p-1$ segments where it is a linear homotopy $A_{s_{j}, j}-(r-2 j+1) Q_{j}, r \in[2 j-1,2 j]$. On the invertible seqments $\operatorname{DF}_{\text {Id }}\left(A_{r}\right)=m_{j} \in \mathbb{Z}, r \in[2 j-2,2 j-1], j=1, \ldots, p$ is constant. By Proposition 8 , we can choose $R_{j} \in \Psi_{\text {sus }}^{-\infty}(Y ; E)$ such that $\operatorname{Id}-R_{j}$ is invertible and $m_{j}=-\sum_{l \leq j} \mathrm{DF}_{\mathrm{Id}}\left(\mathrm{Id}-R_{l}\right)$. Modifying the curve $A_{r}$ successively (in $j$ ) by multiplying on $[2 j-1,2 j]$, by Id $-(r-2 j+1) R_{j}$, and on $[2 j, 2 j+1]$, by Id $-R_{j}$, gives a curve $A_{r}^{\prime}$ with the same properties as before except that $\operatorname{DF}_{\operatorname{Id}}\left(A_{r}^{\prime}\right)$ vanishes on all the 'invertible' intervals $[2 j, 2 j+1]$ and the endpoint has changed to $A \prod_{j}\left(\mathrm{Id}-R_{j}\right)$. Now Proposition 8 can be used again to show, that on each of the intervals $r \in[2 j-1,2 j], A_{r}^{\prime}$ can be deformed to be invertible without changing the endpoints and that the final endpoint can be connected to $A$ by an invertible curve, since

$$
\mathrm{DF}_{\mathrm{Id}}\left(\prod_{j}\left(\mathrm{Id}-R_{j}\right)\right)=0
$$

by assumption. This shows that $A$ is connected to Id by a curve in the invertible set.

This completes the proof of Theorem 4, the surjectivity of

$$
\mathrm{DF}_{\mathrm{Id}}: \mathrm{Ell}_{0}(\mathrm{Id}) \longrightarrow \mathbb{Z}
$$

and hence, the surjectivity in the general case, being a consequence of Proposition 8.

Theorem 3 is an immediate consequence of Theorem 4 . 


\section{References}

1. M. F. Atiyah, V. K. Patodi, and I. M. Singer, Spectral asymmetry and Riemannian geometry, I, Math. Proc. Camb. Phil. Soc 77 (1975), 43-69.

2. J.-M. Bismut and J. Cheeger, Eta-invariants and their adiabatic limits, J.A.M.S. 2 (1989), 33-70.

3. Invent. Math. 89 (1990), 91-151.

4. J.-M. Bismut and D. Freed, The analysis of elliptic families, II, Comm. Math. Phys. 107 (1986), 103.

5. Th. P. Branson and P. B. Gilkey, Residues of the eta function for an operator of Dirac type, J. Funct. Anal. 108 (1992), 47-87.

6. J. Brüning and R. T. Seeley, An index theorem for first order regular singular operators, Amer. J. Math. 110 (1988), 659-714.

7. D. Burns and R. Mazzeo, Private communication.

8. J. Cheeger, Eta invariants, the adiabatic approximation and conical singularities, J. Diff. Geom. 26 (1987), 175-221.

9. R. G. Douglas and K. P. Wojciechowski, Adiabatic limits of the $\eta$-invariants, the odd-dimensional Atiyah-Patodi-Singer problem, Comm. Math. Phys. 142 (1991), 139-168.

10. E. Getzler, Cyclic homology and the Atiyah-Patodi-Singer index theorem, Contemp. Math. 148 (1993), 19-45.

11. M. Lesch and K. P. Wojciechowski, On the $\eta$ invariant of generalized Atiyah-PatodiSinger boundary value problems, Illinois Math. J. (to appear).

12. J. Lott, Higher eta-invariants, K-Theory 6 (1992), 191-233.

13. R. B. Melrose, The Atiyah-Patodi-Singer index theorem, A K Peters, Wellesley, Mass, 1993.

14. R. B. Melrose and P. Piazza, Families of Dirac operators, boundaries and the bcalculus, to appear in J. Diff. Geom., preprint, 1993.

15. An index theorem for families of Dirac operators on odd-dimensional manifolds with boundary, preprint, 1994.

16. W. Müller, Manifolds with cusps of rank one, spectral theory and $L^{2}$-index theorem, Lecture Notes in Math., vol. 1244, Springer Verlag, Berlin, Heidelberg, New York, 1987.

17. _ Eta invariants and manifolds with boundary, J. Diff. Geom. 40 (1994), 311-377.

18. I. M. Singer, The $\eta$-invariant and the index, Mathematical aspects of string theory (S.-T. Yau, ed.), World Scientific, 1988, pp. 239-258.

19. M. Stern, $L^{2}$-index theorems on locally symmetric spaces, Invent. Math. 96 (1989), 231-282.

20. F. Wu, The Chern character and the $\eta$-invariant of the Dirac operator, K-Theory (1993), 145-174.

Department of Mathematics, Mit, Cambridge MA 02139

E-mail address: rbm@math.mit.edu 